\title{
EDITORIAL
}

\section{Read science differently, look at science differently, and publish your science differently}

\author{
Dorel Săndesc ${ }^{1}$ \\ ${ }^{1}$ Faculty of Medicine, "Victor Babes" University of Medicine and Pharmacy, Timisoara, Romania
}

\section{Correspondence to:}

Dorel Săndesc, MD, PhD, Professor, Faculty of Medicine, „Victor Babes” University of Medicine and Pharmacy, Timisoara, Romania

E-mail: dsandesc@yahoo.com

\section{Conflicts of interests \\ Nothing to declare \\ Acknowledgements \\ None}

Central Eur J Clin Res 2018;1(1):1-3

\author{
Published: 27.05.2018
}

Copyright $\odot 2018$ Central European Journal of Clinical Research. This is an open-access article distributed under the Creative Commons Attribution License, which permits unrestricted use, distribution, and reproduction in any medium, provided the original work is properly cited.

Central European Journal of Clinical Research (CEJCR) is a new, open-access, peer-reviewed journal, created by a young and enthusiastic team, that has proven professionalism and passion in the field of clinical research. The editorial board is located in Timisoara, Romania and includes experts in scientific writing: Dorel Sandesc, MD, PhD, Professor (Editor in Chief), Ovidiu Horea Bedreag, MD, PhD, Associate Professor (Assistant Editor), Alexandru Florin Rogobete, MSc, PhD, Clinical Researcher (Executive Editor), Sonia Elena Popovici, MD, BA (Language Editor), and Corina Vernic, PhD, Associate Professor (Statistics Editor). The Editorial Board also includes important figures from all fields of the medical world. The aim of such a multidisciplinary editorial board is to offer a broad expertize to our authors. Hence, CEJCR brings together experts from the fields of emergency medicine, anesthesia and intensive care, surgery, oncology, pediatrics, endocrinology, neurology, dentistry, laboratory medicine, nuclear medicine, genetics, medical chemistry, biochemistry, and biotechnology. CEJCR plans on publishing original articles, review articles, meta-analyses, letters, and case-reports on the most common Clinical Research topics, such as emergency medicine, anesthesia and intensive care, surgery, oncology, pediatrics, endocrinology, neurology, dentistry, laboratory medicine, nuclear medicine, genetics, medical chemistry, biochemistry, and biotechnology. Our aim is to create a top-rated and internationally recognized scientific and educational platform by bringing together the best scientific articles, as well as the best researchers, clinicians, students and experts in the aforementioned fields.

The review process will be carried out in accordance with existing international regulations regarding scientific publishing and with the scientific publishing ethics code. If the received articles comply with the journal's scientific standards, they will be further sent to two different reviewers, both experts in the field. These reviewers will be selected in a double-blind manner. Moreover, the selected reviewers will come from countries different from the country of the authors that sent the manuscript. Following the review process, the articles will be rated as follows: Accepted, Minor Revisions, Major Revi- 
sions, and Rejected. Based on the answer and on the reviewers' decision, the editors can either send the article back to the authors for proofreading or they can reject it definitively.

CEJCR has strict rules regarding the structure of each article. The articles must include the following: (1). Introduction: please state the aims of your paper clearly. In addition, please present a scientific background, that is adequate to the topic of the article and avoid as much as possible excessive details; (2). Material and methods: In this section we kindly ask you to add in enough details in order to make your study/paper reproducible by other authors. If you base your research on methods that were already used and presented in the literature we kindly ask you to cite them accordingly. Furthermore, if you use methods that you have already described in your past articles, please include them as citations; (3). Results: in this section please present the obtained results in a clear, logical and concise manner; (4). Discussions: in this section we kindly ask you to highlight the importance of your study and to discuss the obtained results. Please do not repeat or include the same information presented in the "Results" section. We recommend that you also include here the limitations/biases of your study; (5). Conclusions: please include here the answers to your working hypothesis. It is also advised to present the most significant pro/con arguments regarding the chosen topic; (5). Abstract: the abstract must briefly highlight the aim of the study and the main results. The abstract must also include the summarized conclusions. For Original Papers the Abstract must be structured (Background, Aims, Methods, Results, Conclusions), whiles for other article types it should be unstructured; (6). Keywords: please choose 3-5 keywords that would point out the most important aspects of your study; (7). Abbreviations: please define the abbreviations in the text and also present them as a list at the end of the article; (8). Acknowledgements: this section will appear at the end of the article, before the references section. We recommend that you mention here the persons that brought their contribution to carrying out the study - linguistic editing, proof reading etc. Moreover, we recommend that you introduce a list of funding sources, grants, research projects etc; (9). References: should include the names of all authors when six or fewer. When there are seven or more authors, list only the first six names and add et al., according to Vancouver citation style. Examples: a. Article: Glance LG, Blumberg N, Eaton MP, Lustik SJ, Osler TM, Turner M, et al. Preoperative thrombocytopenia and postoper- ative outcomes after noncardiac surgery. Anesthesiology 2014;120:62-75; b. Book: Pollard BJ. Handbook of clinical anaesthesia. 3rd edition. London: Hodder Arnold, 2011; c. Chapter in books: Naguib M, Lien CA. Pharmacology of muscle relaxants and their antagonists. In: Miller RD, Eriksson LI, Fleisher LA, Wiener Kronish JP, Young WL, editors. Miller's Anesthesia. 7th edition. New York: Churchill Livingstone, 2010:859-912.

Submitting your scientific paper for publication in Central European Journal of Clinical research implies that you confirm, in an Authorship Statement, the following: the paper is original and it has never been published in its current form or a substantially similar form (with the exception of papers that have been published previously as abstracts or parts of a course study or a PhD thesis); it has not been accepted for publication by another journal, and it is not under consideration by another publication.

All authors will be asked to sign a form confirming that they have read and approved with the paper. Papers are accepted for publication with the understanding that exclusive copyright of the paper is assigned to Central European Journal of Clinical Research and Clinical Research Network S.R.L.. The assignment becomes effective when the article has been accepted for publication. Editors will check every submitted manuscript for plagiarism. At the editors' or reviewers' request, the authors should be able to offer the raw data of their study submitted for publication.

\section{Ethics in publishing}

Scientific misconduct includes plagiarism, data fabrication, falsification, ghost authorship or other fraudulent research practices. In order to prevent plagiarism issues, all manuscripts sent for review will be screened using the plagiarism detection software.

In case of the supposition of scientific misconduct, the Editor will act in accordance with the procedure specified in the COPE guidelines and if the academic fraud is confirmed the manuscript will be rejected for publication.

For all research studies involving human subjects the author must receive approval of the Hospital or University Ethics Committee, as well as a written, informed consent from all the patients included in the study; this fact must be mentioned in the Methods section. Lack of approval from the Ethics Committee and/or the absence of the patient's informed consent will result in the rejection of the manuscript. 


\section{Human and animal rights}

Studies involving experimental research on either animals or humans must conform to the principles mentioned in the Declaration of Helsinki. So as respect the patient's right to privacy, information such as patient's name, photos, should not be part of any published material. In order to publish photographs or other materials that could be used in identifying one patient, the author must be granted permission by attaching a copy of the written patient's consent form. Manuscripts reporting experimental data based on animal studies should include specific information in accordance to ARRIVE (Animal Research: Reporting In Vivo Experiments) guidelines. (http://www.nc3rs.org.uk/downloaddoc. asp? $i d=1206 \&$ page $=1357 \&$ skin=0)

\section{Conflict of interest}

All received articles should include a statement regarding the conflict of interest, both financial or of a different nature. If one or more authors have any kind of conflict of interest they must be declares. If there is no type of conflict of interest this should be stated as "Nothing to declare".

Hence, we are taking this road together, with an ambitious project that wishes to bring to the international community's attention the best scientific articles, as well as to unite researchers and clinicians worldwide under the roof of this new scientific platform. We want to believe that CEJCR will represent a new era in the field of scientific publishing.

In order to achieve this purpose we need help to make this journal "grow", a goal that can only be achieved together, with your valuable contributions. We want to kindly ask you to contribute together with your research groups and PhD students with scientific articles for the following issues of the journal.

The first number will be published in September and launched at the International Conference for Guidelines and Protocols in Anaesthesia, Intensive Care and Emergency Medicine 2018, that will be held in Chisinau, Republic of Moldavia.

CEJCR has successfully passed all the verification steps of the DeGruyter Ltd editorial house, and following this recognition will be assigned a separate page in their search engine. Moreover, the journal will be indexed starting with the first issue in numerous international databases (Scopus, ProQuest, WorldCat, DOI).

We plan to begin the verifications requested by PubMed Central in January 2019, while the verifications for Thomson Reuters (Analytics) will start at the end of next year. All these steps that we are willing to take are only made possible by your interest and professionalism and therefore we would like to thank all of you for the support. 


\title{
LETTER TO EDITOR
}

\section{Recurrent Wheezing}

\author{
Ioana M.Ciuca ${ }^{1,2}$, Elena Margau ${ }^{2,3}$ \\ ${ }^{1}$ Pediatric Department, University of Medicine and Pharmacy "Victor Babes" Timisoara, \\ Timisoara, Romania \\ ${ }^{2}$ Clinic II Pediatric, Clinical County Hospital Timisoara, Timisoara, Romania \\ ${ }^{3}$ Pediatric Hospital Louis Turcanu, Timisoara, Romania
}

\section{Correspondence to:}

Assistant professor Oana M.Ciuca, PhD, Pediatric Department, University of Medicine and Pharmacy" Victor Babes" Timisoara; Clinic II Pediatric, Clinical County Hospital, Timisoara, Romania

Contact address: Romania, Timisoara, Evlia Celebi street 1-3

E-mail: ciuca.ioana@umft.ro

\section{Conflicts of interests}

Nothing to declare

\section{Acknowledgment}

None

Funding: This research did not receive any specific grant from funding agencies in the public, commercial or not-for profit sectors.

Keywords: atrial fibrillation; quality of life; comorbidities; anticoagulants; mortality.

These authors take responsibility for all aspects of the reliability and freedom from bias of the data presented and their discussed interpretation.

Central Eur J Clin Res 2018;1(1):4-8

Received: 15.06.2018, Accepted: 13.08.2018, Published: 5.09.2018

Copyright $\odot 2018$ Central European Journal of Clinical Research. This is an open-access article distributed under the Creative Commons Attribution License, which permits unrestricted use, distribution, and reproduction in any medium, provided the original work is properly cited.

\section{Introduction}

Wheezing is the most frequent clinical finding in pediatric pulmonary disorders, approximately $30 \%$ of children manifest an episode of wheezing during their lives [1,2].

Recurrent wheezing, demonstrated by repeating attacks of wheezes is a common complaint in pediatric everyday practice. Having a high chance to happen, the progression into asthma in some cases with recurrent wheezing lay down a possibility of early treatment modalities before developpment of pulmonary remod- elling phenomenon and deterioration of pulmonary functions [3].

Accordingly, noticable discussions have erupted regarding the classification and treatment of preschoolers complaining of wheezing episodes and in order to wrap them up, the primary aetiology and pathophysiological mechanisms of these episodes have to be accurately explained $[1,4,5]$.

\section{Epidemiology}

Recurrent wheeze and dyspnea in preschool children was studied in more cohort studies on a large-scale age. As a landmark study 